\title{
Tick capillary feeding for the study of proteins involved in tick-pathogen interactions as potential antigens for the control of tick infestation and pathogen infection
}

Sandra Antunes ${ }^{1 \dagger}$, Octavio Merino ${ }^{2 \dagger}$, Juan Mosqueda ${ }^{3}$, Juan A Moreno-Cid ${ }^{2}$, Lesley Bell-Sakyi ${ }^{4,5}$, Rennos Fragkoudis ${ }^{4,5}$, Sabine Weisheit ${ }^{4,5}$, José M Pérez de la Lastra ${ }^{2}$, Pilar Alberdi ${ }^{2}$, Ana Domingos ${ }^{6}$ and José de la Fuente ${ }^{2,7^{*}}$

\begin{abstract}
Background: Ticks represent a significant health risk to animals and humans due to the variety of pathogens they can transmit during feeding. The traditional use of chemicals to control ticks has serious drawbacks, including the selection of acaricide-resistant ticks and environmental contamination with chemical residues. Vaccination with the tick midgut antigen BM86 was shown to be a good alternative for cattle tick control. However, results vary considerably between tick species and geographic location. Therefore, new antigens are required for the development of vaccines controlling both tick infestations and pathogen infection/transmission. Tick proteins involved in tick-pathogen interactions may provide good candidate protective antigens for these vaccines, but appropriate screening procedures are needed to select the best candidates.

Methods: In this study, we selected proteins involved in tick-Anaplasma (Subolesin and SILK) and tick-Babesia (TROSPA) interactions and used in vitro capillary feeding to characterize their potential as antigens for the control of cattle tick infestations and infection with Anaplasma marginale and Babesia bigemina. Purified rabbit polyclonal antibodies were generated against recombinant SUB, SILK and TROSPA and added to uninfected or infected bovine blood to capillary-feed female Rhipicephalus (Boophilus) microplus ticks. Tick weight, oviposition and pathogen DNA levels were determined in treated and control ticks.

Results: The specificity of purified rabbit polyclonal antibodies against tick recombinant proteins was confirmed by Western blot and against native proteins in tick cell lines and tick tissues using immunofluorescence. Capillary-fed ticks ingested antibodies added to the blood meal and the effect of these antibodies on tick weight and oviposition was shown. However, no effect was observed on pathogen DNA levels.

Conclusions: These results highlighted the advantages and some of the disadvantages of in vitro tick capillary feeding for the characterization of candidate tick protective antigens. While an effect on tick weight and oviposition was observed, the effect on pathogen levels was not evident probably due to high tick-to-tick variations among other factors. Nevertheless, these results together with previous results of RNA interference functional studies suggest that these proteins are good candidate vaccine antigens for the control of $R$. microplus infestations and infection with A. marginale and B. bigemina.
\end{abstract}

Keywords: Tick, Pathogen, Anaplasma, Babesia, Capillary feeding, Vaccine, Tick cell line

\footnotetext{
* Correspondence: jose_delafuente@yahoo.com

${ }^{\dagger}$ Equal contributors

${ }^{2}$ SaBio. Instituto de Investigación en Recursos Cinegéticos

IREC-CSIC-UCLM-JCCM, Ronda de Toledo s/n, 13005 Ciudad Real, Spain

${ }^{7}$ Department of Veterinary Pathobiology, Center for Veterinary Health

Sciences, Oklahoma State University, Stillwater, OK 74078 USA

Full list of author information is available at the end of the article
} 


\section{Background}

Ticks are hematophagous ectoparasites whose feeding can have an adverse effect on animal health and production [1]. In particular, Rhipicephalus (Boophilus) spp. cattle ticks affect weight gain and milk production and transmit pathogens that cause bovine anaplasmosis (Anaplasma marginale) and babesiosis (Babesia bigemina and Babesia bovis) in tropical and subtropical regions of the world $[2,3]$. Chemical acaricides are currently the main method for the control of tick infestations, but problems associated with their use, such as limited efficacy in some regions due to selection of acaricide-resistant ticks and contamination of the environment and animal products with chemical residues, indicate the need for alternative control methods [4].

A promising alternative for the control of tick-borne diseases is vaccination with recombinant tick antigens [5]. Vaccination with commercial vaccines containing the recombinant Rhipicephalus (Boophilus) microplus BM86 gut antigen demonstrated their advantages for tick control, including cost-effectiveness and reduction in acaricide application. In addition, these vaccines also reduced the prevalence of anaplasmosis and babesiosis in some regions, presumably through reducing exposure of cattle to infected ticks [6]. However, BM86-based vaccines have variable efficacy against different geographic strains of $R$. microplus and do not affect tick vector capacity [6]. Therefore, new antigens are required for the development of vaccines affecting tick feeding, reproduction and vector capacity to control both tick infestations and pathogen infection/transmission.

Recently, the application of molecular biology approaches including transcriptomics and proteomics to the characterization of interactions between ticks (Rhipicephalus spp.) and pathogens (A. marginale or B. bigemina), with functional analyses using RNA interference (RNAi), have resulted in the identification of tick proteins with a possible role in pathogen infection and transmission [7-11]. However, additional analyses are required to characterize the potential of these proteins as vaccine antigens for the control of tick infestations and pathogen infection [12].

One approach to increase the possibilities of identifying tick protective antigens is to combine RNAi functional studies with in vitro tick feeding. RNAi allows screening of a relatively large number of genes involved in tickpathogen interactions, while in vitro feeding with antibodies against selected candidate antigens should provide results more closely resembling vaccine protective capacity [12]. In vitro tick feeding techniques have been used for studies on tick biology and tick-pathogen interactions [13-19] and more recently to test the effect on tick feeding of antibodies added to the blood meal [20-22].

In the present study, we selected proteins involved in interactions between tick and A. marginale (Subolesin
(SUB) and SILK) $[7,9,10]$ and between tick and B. bigemina (TROSPA) [11], as determined by systems biology and RNAi functional studies, to characterize their potential as antigens for the control of both $R$. microplus tick infestations and infection with A. marginale or B. bigemina using in vitro capillary feeding with purified rabbit polyclonal antibodies against recombinant proteins. The results showed the possibilities and some of the limitations of this approach for the identification of candidate tick protective antigens.

\section{Methods}

\section{Experimental design and rationale}

The objective of this research was to evaluate the possibilities of identifying tick protective antigens using in vitro tick feeding with antibodies directed against tick proteins involved in tick-pathogen interactions, as determined by systems biology and RNAi functional studies. Antibodies were produced in rabbits using $R$. microplus recombinant proteins involved in tick- $A$. marginale (Subolesin (SUB) and SILK) $[7,9,10]$ and tick-B. bigemina (TROSPA) [11] interactions. Partially engorged female $R$. microplus ticks were capillary fed with purified rabbit polyclonal antibodies against recombinant proteins added to blood collected from cattle uninfected and infected with $A$. marginale or $B$. bigemina. After in vitro capillary feeding, the effect of the treatments on tick weight, oviposition and pathogen DNA levels was measured as indicators of the potential of these antigens for the control of both $R$. microplus tick infestations and pathogen infection. Animal experiments were carried out in strict accordance with the Guide for Care and Use of Laboratory Animals for the University of Queretaro and the protocol was approved by the Committee on the Ethics of Animal Experiments (Permit no.: 23FCN2012).

\section{Expression and purification of recombinant proteins}

The R. microplus SUB (Genbank accession number GQ456170.1) coding region was amplified by RT-PCR using oligonucleotides 5'-CACCATGGCGTGCGCCACC CTGAAAC-3' and 5'-TTAAGACAGATAAGACGGG GTG-3' and total RNA from the acaricide-susceptible and Anaplasma- and Babesia-free Media Joya strain, CENAPA, Mexico [23-25]. The genes encoding Rhipicephalus (Boophilus) annulatus TROSPA (JK489429) and R. microplus SILK (GO496219) proteins were synthesized by GenScript (Piscataway, NJ, USA). Tick genes were cloned into the expression vector $\mathrm{pET101/D-TOPO}{ }^{\circ}$ (Invitrogen, Carlsbad, CA, USA) following the manufacturer's recommendations. Recombinant constructs were transformed into $\mathrm{Bl} 21$ Escherichia coli cells (Invitrogen, Carlsbad, CA, USA) and inoculated into Luria broth containing $50 \mathrm{mg} / \mathrm{ml}$ ampicillin and $0.4 \%$ glucose. Cultures were grown at $37^{\circ} \mathrm{C}$ to an $\mathrm{OD}_{600 \mathrm{~nm}}=0.4$. Isopropyl- $\beta$-d-thiogalactopyranoside 
(IPTG) was then added to a final concentration of $0.5 \mathrm{mM}$ and incubated for $4 \mathrm{~h}$ to induce the production of recombinant proteins. Cells were collected by centrifugation and recombinant proteins were purified to $80-90 \%$ purity by $\mathrm{Ni}$ affinity chromatography using the Ni-NTA Spin kit (QIAGEN, Hilden, Germany). Protein concentration was measured using the Pierce ${ }^{\circ}$ BCA protein assay kit (Thermo Scientific, Rockford, IL, USA). Purified proteins were analyzed by SDS-PAGE and Western blot.

\section{Production of polyclonal antibodies}

For each tick protein, three New Zealand white rabbits (Oryctulagus cuniculus) were subcutaneously injected at weeks 0,3 and 6 with $50 \mu \mathrm{g}$ protein in $0.5 \mathrm{ml}$ Montanide ISA $50 \mathrm{~V}$ adjuvant (Seppic, Paris, France). Blood was collected before injection and two weeks after the last immunization to prepare preimmune and immune sera, respectively. Serum aliquots were kept at $4^{\circ} \mathrm{C}$ for immediate use or at $-20^{\circ} \mathrm{C}$ for long-term storage. IgGs were purified from serum samples using the Montage Antibody purification kit and spin columns with PROSEP-A Media (Millipore, MA, USA) following the manufacturer's recommendations.

\section{Western blot analysis}

Ten micrograms of each protein were loaded into a $10 \%$ SDS-polyacrylamide pre-cast gel (Expedeon, San Diego, CA, USA). Samples were electrophoresed for $1 \mathrm{~h}$ at $180 \mathrm{~V}$ constant current. The SDS-PAGE gel was transferred to a nitrocellulose membrane (Whatman, Dassel, Germany) during $1 \mathrm{~h}$ at $12 \mathrm{~V}$. The membrane was blocked with $5 \%$ skimmed milk in TBS for $1 \mathrm{~h}$ at room temperature, washed three times in TBS and incubated overnight with $2.5 \mu \mathrm{g} / \mathrm{ml}$ of the purified polyclonal IgGs in TBS, then washed three times with TBS and incubated with an anti-rabbit horseradish peroxidase (HRP) conjugate (Sigma-Aldrich, St. Louis, MO, USA) diluted 1:1000 in TBS. The membrane was washed three times with TBS and finally developed with 3, 3', 5, 5'-tetramethylbenzidene (TMB) stabilized substrate for HRP (Promega, Madison WI, USA).

\section{Tick cell lines}

Three embryo-derived tick cell lines, $R$. microplus BME/ CTVM2 [26] and Ixodes scapularis IDE8 [27] and ISE6 [28] were used in the experiment. BME/CTVM2 cells were cultured in L-15 (Leibovitz) medium supplemented with $10 \%$ tryptose phosphate broth (TPB) and $20 \%$ fetal calf serum (FCS) at $28^{\circ} \mathrm{C}$. IDE8 and ISE6 cells were grown in L-15B medium [29] supplemented with $10 \%$ TPB, 5\% FCS and $0.1 \%$ bovine lipoprotein concentrate (MP Biomedicals, UK) at $32^{\circ} \mathrm{C}$. Both media were supplemented with $2 \mathrm{mM}$ L-glutamine, $100 \mathrm{IU} / \mathrm{ml}$ penicillin and $100 \mu \mathrm{g} / \mathrm{ml}$ streptomycin. Tick cell lines were maintained in flat-sided tubes (Nunc). Medium changes were carried out weekly by removal and replacement of twothirds of the medium volume. Cultures were passaged at a split ratio of 1:1 at 2-3 week intervals as follows: an equal volume of fresh medium was added to the parent tube, cells were resuspended by gentle pipetting, and half the resultant cell suspension was transferred to a new culture tube (previously conditioned by incubating fresh culture medium therein for several hours) while leaving the remainder in the parent tube for reattachment.

\section{Immunofluorescence in tick cells}

For the immunofluorescence assay, tick cells were harvested by pipetting, counted by haemocytometer and seeded at $5-6 \times 10^{5}$ cells $/ \mathrm{ml}$ in $1 \mathrm{ml}$ fresh medium onto glass coverslips inside 24-well plates. After overnight incubation the cells were fixed in situ with $10 \%$ neutral buffered paraformaldehyde for $1 \mathrm{~h}$. After a wash with PBS, the cells were permeabilized by covering with $0.3 \mathrm{ml}$ Triton $\mathrm{X}-100 / \mathrm{PBS}$ for $30 \mathrm{~min}$. The Triton $\mathrm{X}-100 / \mathrm{PBS}$ was removed and the cells were coated with $0.1 \%$ SDS/PBS for $10 \mathrm{~min}$. The cells were then washed with PBS and blocked with CAS-block (Invitrogen, Paisley, UK) for $60 \mathrm{~min}$ and then incubated overnight at $4{ }^{\circ} \mathrm{C}$ with the purified IgGs diluted 1:100 in CAS-block. After at least $3 \times 5$ min additional washes with PBS, they were incubated in CASblock containing a secondary FITC-conjugated anti-rabbit antibody (1:500; AbD Serotec, Oxford, UK) for $1 \mathrm{~h}$ at room temperature. Cells were washed three more times with PBS and the coverslips were drained and mounted onto microscope slides using Vectashield hardset mounting medium (Vector Laboratories, Peterborough, UK). For fluorescence and light microscopy, a confocal microscope was used (Zeiss AxioSkop confocal microscope; Carl Zeiss Ltd., Welwyn Garden City, UK) and images were analyzed with Zeiss Zen software.

\section{Immunofluorescence in tick tissues}

Tick guts, salivary glands and ovaries were dissected in ice-cold PBS from individual engorged $R$. microplus females. All tissues were washed in PBS and the luminal content was carefully removed from the guts. Tissues were either used immediately after dissection or stored at $-80^{\circ} \mathrm{C}$ in RNAlater (Ambion, Austin, TX, USA) until use. To prepare samples for indirect fluorescence microscopy, dissected tissues were placed in small plastic cassettes, fixed in $3.7 \%$ formaldehyde, dehydrated in increasing ethanol dilutions and infiltrated with paraffin (Histosec, Merck, Whitehouse Station, NJ, USA). After cooling, tissues were sectioned using a SM2010 R sliding microtome (Leica, Carnaxide, Portugal). The 2-3 $\mu \mathrm{m}$ sections were placed on a glass slide, allowed to dry and then subjected to deparaffinization and dewaxing in xylene and hydrated in decreasing ethanol concentrations. Tissues were then permeabilized with $0.5 \%$ Triton X-100 
(v/v) in PBS for 30 min and washed three times with excess PBS before blocking overnight with $3 \%$ BSA (w/v) at $4^{\circ} \mathrm{C}$. After washing the slides again with PBS, solutions of purified IgGs diluted 1:100 in blocking solution were applied and incubated for $1 \mathrm{~h}$ at $37^{\circ} \mathrm{C}$. The slides were then washed three times with $\mathrm{PBS}$ and the secondary antibody Alexa Fluor 488 (green)-conjugated anti-rabbit antibody (Molecular Probes, Life Technologies, Porto, Portugal) diluted 1:100 in blocking solution was applied and slides incubated for $1 \mathrm{~h}$ at $37^{\circ} \mathrm{C}$. After a final PBS wash, a drop of ProLong ${ }^{\bullet}$ Gold Antifade Reagent with 4',6'-diamidino-2-phenylindole (DAPI) (Invitrogen) was placed over the sections and then sealed with a coverslip. Slides were kept in a moist dark box until microscopic analysis to prevent drying and fluorescence fading. Tick tissue sections were visualized under a Nikon Eclipse $80 i$ fluorescence microscope with appropriate filters (Nikon Instruments Inc., Barcelona, Spain).

\section{Tick capillary feeding}

To obtain B. bigemina and A. marginale infected blood, two 6 month-old Babesia/Anaplasma-free calves were splenectomized and two weeks later intravenously inoculated one each with cryopreserved $1 \times 10^{6} \mathrm{~B}$. bigemina (Chiapas strain; [30]) or $1 \times 10^{8}$ A. marginale (Morelos strain; [31]) infected erythrocytes (IE). After inoculation, animals were regularly monitored by checking their rectal temperature and hematocrit, and by examination of Giemsa-stained blood smears. Once the parasitemia levels reached $0.7 \%$ IE, $500 \mathrm{ml}$ blood was collected into collection bags containing $70 \mathrm{ml}$ citrate phosphate dextrose anticoagulant. Uninfected bovine blood was obtained in a similar manner from a healthy calf.

Tick artificial feeding was carried out with partially engorged female $R$. microplus (Media Joya strain) ticks recovered manually from calves 20-21 days after infestation with larvae. Collected ticks were cleaned, weighed and fixed on expandable polystyrene plates $(19 \times 10 \mathrm{~cm})$ with double-sided adhesive tape (3 M, St. Paul, MN, USA). Female ticks were discarded if they had damaged mouthparts or their weight did not lie between 20 and $60 \mathrm{mg}$. Citrated bovine blood from uninfected or infected animals was used to fill microhematocrit capillary tubes $(75 \times \varnothing 1.5 \mathrm{~mm})$ that were placed over the ticks' mouthparts. Tubes were replaced every $2-3 \mathrm{~h}$, as described previously [22]. Female ticks were divided into experimental groups of 15 individuals each and fed for $28 \mathrm{~h}$ with uninfected, $B$. bigemina-infected or $A$. marginaleinfected blood alone or supplemented with $1 \mathrm{mg} / \mathrm{ml}$ of preimmune or antigen-specific purified IgGs. After feeding, ticks were detached from the double-sided tape and weighed again to determine tick weight increase during feeding. Five ticks per group were then placed in Petri dishes and incubated at $27^{\circ} \mathrm{C}$ and $85 \%$ humidity for oviposition [7]. Weight increase during feeding (mg/tick) and oviposition (weight of eggs/tick in mg) were compared between ticks fed with blood supplemented with antibodies against the selected recombinant proteins and control ticks fed with bovine blood supplemented with preimmune antibodies by Student's $t$ test with unequal variance $(\mathrm{P}=0.05)$.

This study was carried out in strict accordance with the Guide for Care and Use of Laboratory Animals for the University of Queretaro and the protocol was approved by the Committee on the Ethics of Animal Experiments (Permit no.: 23FCN2012).

\section{PCR to determine pathogen DNA levels in ticks}

In order to quantify pathogen DNA levels, 10 ticks per group were incubated in Petri dishes at $27^{\circ} \mathrm{C}$ and $85 \%$ humidity for three days after feeding and their internal tissues dissected to obtain total RNA and DNA using TRI Reagent (Invitrogen, Carlsban, CA, USA) following the manufacturer's recommendations. For each tick, A. marginale and $B$. bigemina DNA levels were analyzed by real-time PCR using the oligonucleotide primers RTMSP4F 5' -GACGTG CTGCACACAGATTT-3'/RTMSP4R 5'-CTCATCAAAT AGCCCGTGGT-3' and RTBbF 5'-AGCTTGCTTTCA CAACTCGCC-3'/RTBbR 5'-TTGGTGCTTTGACCGAC GACAT-3' to amplify the A. marginale major surface protein 4 (msp4) (AF428083) and B. bigemina 16S rDNA (HQ264118) genes, respectively. Real-time PCR was performed using the QuantiTec SYBR Green RT-PCR kit (Qiagen, Valencia, CA, USA) and a Bio-Rad iQ5 thermal cycler following the manufacturer's recommendations. The A. marginale msp4 and B. bigemina $16 \mathrm{~S}$ rDNA levels were normalized against tick $16 \mathrm{~S}$ rDNA using the comparative Ct method [32]. Pathogen DNA levels in ticks (arbitrary units) were compared between ticks fed with blood supplemented with antibodies against the selected recombinant proteins and control ticks fed with bovine blood supplemented with preimmune antibodies by Student's test with unequal variance $(\mathrm{P}=0.05)$.

\section{Characterization of tick mRNA levels of genes encoding for vaccine antigens}

The sub, silk and trospa mRNA levels were characterized in 10 ticks per group by real-time RT-PCR [33] using the oligonucleotide primers SubolrtFw:5'-CACAGTCCGAGT GGCAGAT-3' and SubolrtRev:5'-GATGCACTGGTGAC GAGAGA-3', SilkrtFw:5'-GGACGTCAATCCGCATT TGG-3' and SilkrtRev:5'-GGCCATAGCCGTAGTTT CCA-3', TROSPArtFw:5' -AGGTTACGGACACGGAG GA-3' and TROSPArtRev:5' -GCCCAAGCGCATAAATAAGA-3' for sub, silk and trospa, respectively. Realtime RT-PCR was performed using the QuantiTec SYBR Green RT-PCR kit (Qiagen, Valencia, CA, USA) and a Bio-Rad iQ5 thermal cycler (Hercules, CA, USA) 
following the manufacturer's recommendations. The mRNA levels were normalized against tick $16 \mathrm{~S}$ rRNA using the comparative $\mathrm{Ct}$ method [32]. Normalized mRNA levels were compared between ticks fed with blood supplemented with antibodies against the selected recombinant proteins and control ticks fed with bovine blood supplemented with preimmune antibodies by Student's $t$ test with unequal variance $(P=0.05)$.

\section{Results}

Production and characterization of antibodies against tick proteins involved in tick-pathogen interactions

Proteins involved in tick- $A$. marginale (Subolesin (SUB), SILK) and tick- $B$. bigemina (TROSPA) interactions as determined by systems biology and RNAi functional studies were selected to characterize their potential as antigens for the control of both $R$. microplus tick infestations and infection with $A$. marginale or $B$. bigemina. Recombinant proteins were produced in $E$. coli (Figure 1A) and used to obtain polyclonal antibodies in rabbits for in vitro capillary tick feeding experiments.

The antibodies were first characterized by Western blot showing the specific recognition of their respective denatured antigens (Figure 1B). The antibodies were then characterized for their ability to recognize tick proteins by immunofluorescence in cultured tick cells (Figure 2A-H) derived from both homologous $(R$. microplus) (Figure 2A-E) and heterologous (I. scapularis) (Figure 2F and G) species and in $R$. microplus fed female guts, salivary glands and ovaries (Figure 3A-D). Preliminary analysis showed that all antibodies recognized tick proteins in different cellular compartments in the tick cell lines and tissues. For example, antibodies against SILK (Figures 2A, B and 3A) and TROSPA (Figures 2C, D and 3B) preferentially recognized intracellular structures and cell membrane, respectively. Anti-SUB antibodies recognized proteins in the nucleus, perinuclear region and cytoplasm of tick cells (Figures 2E-G and 3C). Antibodies against $R$. microplus SUB were tested and showed positive reactions in both $R$. microplus (BME/CTVM2) and I. scapularis (IDE8 and ISE6) cell lines.

\section{Effect of antibodies against tick proteins on tick weight and oviposition and pathogen infection}

Capillary feeding experiments were conducted to evaluate the effect of antibodies against selected tick proteins on tick weight and oviposition. When ticks were fed with uninfected bovine blood supplemented with antibodies against tick proteins, only the group with anti-SUB IgGs showed a significant reduction in tick weight (24\% reduction; $\mathrm{P}=0.0003)$ when compared to ticks fed on blood with preimmune serum (Figure 4A). When ticks were fed on B. bigemina-infected blood, only groups with antiTROSPA and anti-SUB IgGs showed reductions in tick weight, of $18 \%(\mathrm{P}=0.04)$ and $37 \%(\mathrm{P}=0.001)$ respectively, when compared to ticks fed on blood with preimmune serum (Figure 4B). When ticks were fed on $A$. marginaleinfected blood, tick weight was similar between all groups (Figure 4C).

Oviposition was reduced when compared to ticks fed on blood supplemented with preimmune antibodies in the groups fed on uninfected blood with antiSILK (62\% reduction; $\mathrm{P}=0.005)$ and anti-SUB (70\% reduction; $\mathrm{P}=0.001$ ) antibodies (Figure $5 \mathrm{~A}$ ) and on
A

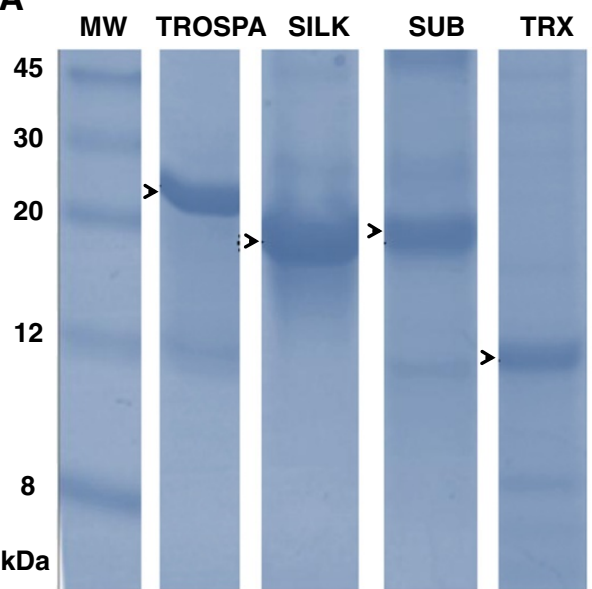

B

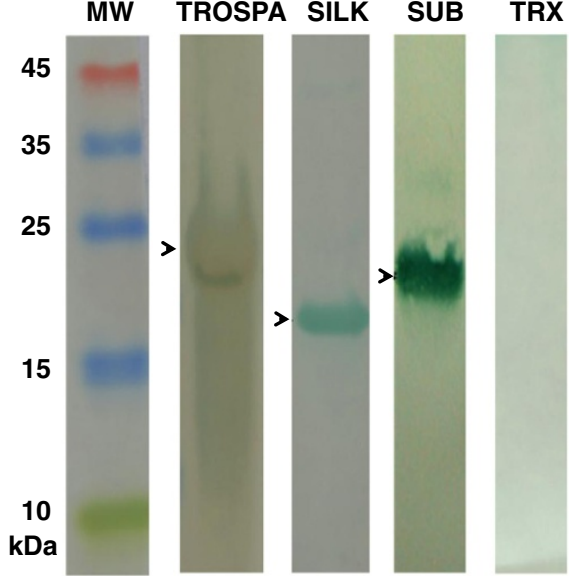

Figure 1 Production of recombinant proteins and antibodies. (A) Ten micrograms of recombinant proteins TROSPA, SILK, Subolesin (SUB) and thioredoxin (TRX; negative control) were loaded onto an SDS-PAGE gel under reducing conditions and visualized by Coomassie brilliant blue staining. (B) Western blot analysis of the recombinant proteins using purified lgGs produced in rabbits immunized with these proteins. Hybridization signals were developed with an anti-rabbit IgG HRP conjugate and TMB substrate for HRP. Arrows indicate the position of recombinant proteins. Abbreviation: MW, molecular weight. 

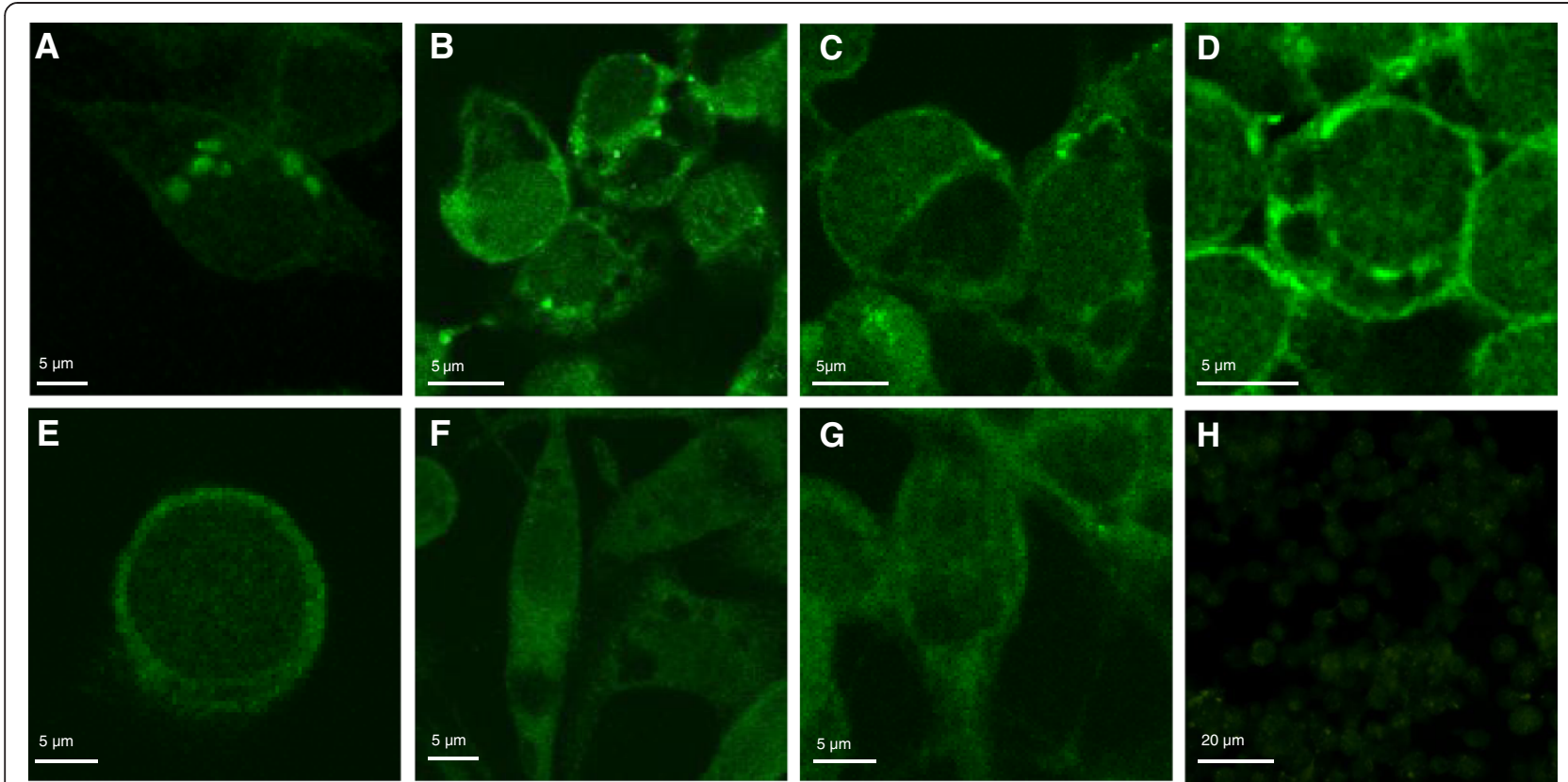

Figure 2 Immunofluorescence analysis of tick cells. Representative images of imunofluorescence analysis of tick cells. Tick cells were stained with rabbit anti-tick protein antibodies (green, FITC). (A, B) BME/CTVM2 cells stained with anti-SILK antibodies. (C, D) BME/CTVM2 cells stained with anti-TROSPA antibodies. (E) BME/CTVM2 cells stained with anti-SUB antibodies. (F) IDE8 cells stained with anti-SUB antibodies. (G) ISE6 cells stained with anti-SUB antibodies. (H) preimmune control serum-treated BME/CTVM2 cells. Scale bars, $5 \mu \mathrm{m}(\mathbf{A}-\mathbf{G}) ; 20 \mu \mathrm{m}(\mathbf{H})$.

B. bigemina-infected blood with anti-SUB IgGs $(43 \%$ reduction; $\mathrm{P}=0.01$ ) (Figure $5 \mathrm{~B}$ ). No reduction in oviposition was observed in ticks fed on $A$. marginale-infected blood supplemented with antibodies against tick proteins (Figure 5C).

\section{Effect of antibodies against tick proteins on tick pathogen infection}

Capillary feeding experiments with antibodies against selected tick proteins were conducted to evaluate the effect on tick infection with $B$. bigemina and $A$. marginale. Pathogen DNA levels in ticks fed on B. bigemina-infected blood (Figure 6A) but not in ticks fed on A. marginale-infected blood (Figure 6B) were significantly higher when compared to ticks fed on uninfected blood ( $\mathrm{p}<0.001$; data not shown). However, significant differences in pathogen DNA levels at day 3 after feeding were not found between groups fed on blood supplemented with preimmune and immune IgGs (Figure 6A and B).

Effect of antibodies and pathogen infection on the mRNA levels of genes encoding for tick proteins

The mRNA levels of genes encoding for selected tick proteins were characterized in ticks fed on uninfected and infected blood (Figure 6C). The results showed no difference in gene expression levels between ticks fed on uninfected and infected blood (Figure 6C). Because of the role of SUB as a transcription factor involved in its own regulation, sub mRNA levels were characterized in ticks fed on blood supplemented with preimmune and anti-SUB IgGs (Figure 6D). The results showed that antiSUB IgGs reduced sub expression in ticks when compared to ticks fed on uninfected blood (67\% reduction; $\mathrm{P}=0.05$ ) and blood supplemented with preimmune IgGs (90\% reduction; $\mathrm{P}=0.01$ ) (Figure 6D).

\section{Discussion}

SUB

Antibodies against $R$. microplus SUB reacted positively in R. microplus BME/CTVM2 cells and tissues and in $I$. scapularis IDE8 and ISE6 cell lines, supporting SUB ubiquitous expression and amino acid sequence conservation in ticks [34]. Vaccination with SUB has shown an effect on the control of $R$. microplus infestations and infection with $A$. marginale and B. bigemina $[33,35]$. In the present study, as in previous experiments with vaccinated cattle [36], anti-SUB antibodies reduced tick weight and oviposition in ticks fed on uninfected blood. However, while tick weight and oviposition were affected by anti-SUB antibodies when ticks were fed on $B$. bigemina-infected blood, no effect was seen with $A$. marginale-infected blood, in contrast to the results of a previous vaccination trial in cattle [33,35]. Additionally, A. marginale and B. bigemina DNA levels did not differ in ticks fed on infected blood with anti-SUB antibodies when compared to ticks fed on blood supplemented with preimmune serum, again showing differences from the results of the vaccination trial in cattle $[33,35]$. Additionally, the expression of sub has been 


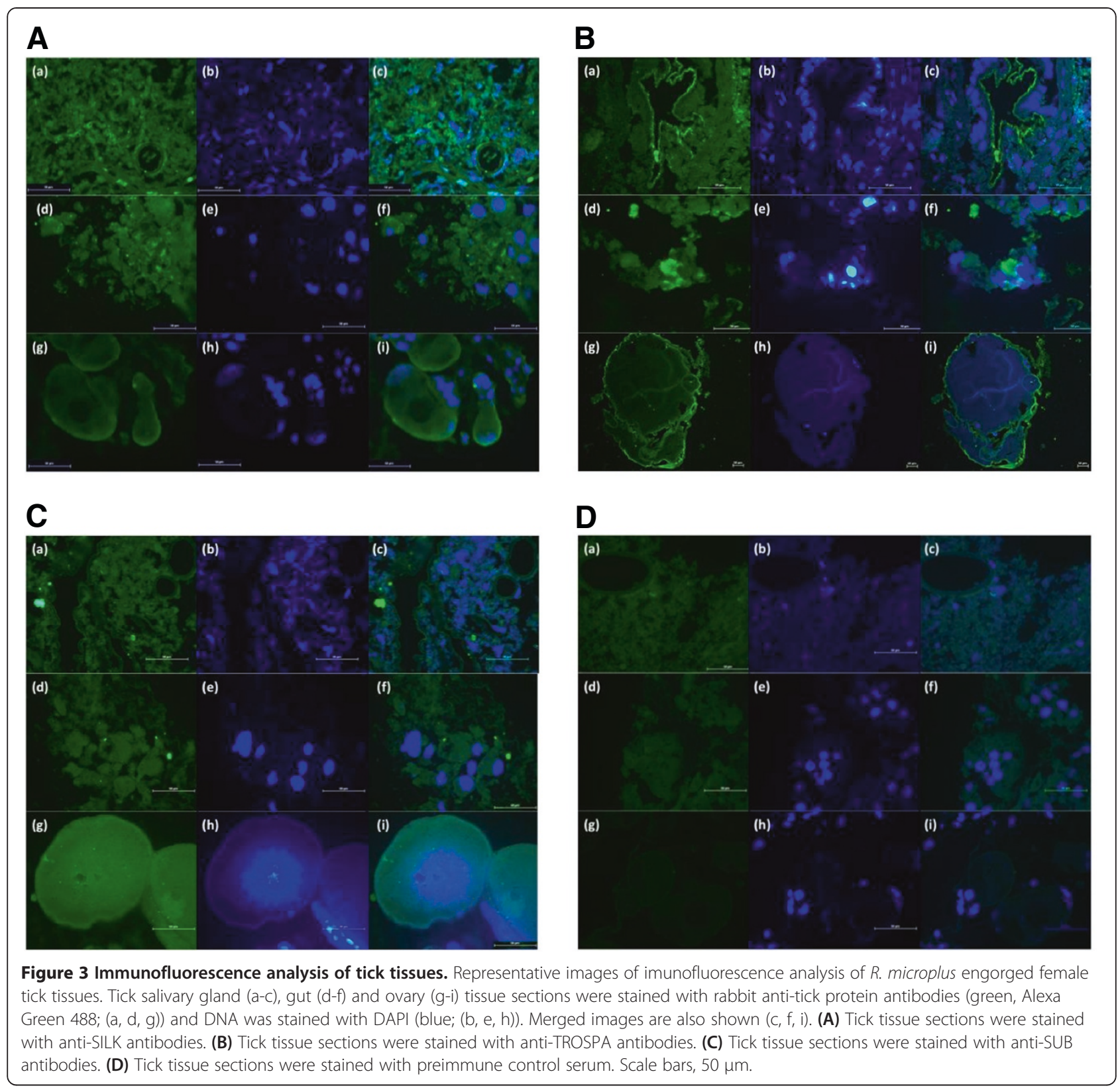

shown to increase in response to $A$. marginale and $B$. bigemina infection in ticks $[33,35]$. However, in capillaryfed ticks, sub mRNA levels did not increase in response to infection. Nevertheless, the reduction in $s u b$ mRNA levels in ticks fed on uninfected blood with anti-SUB antibodies suggested that ticks did ingest antibodies in a manner resembling feeding on vaccinated cattle $[33,35,36]$.

SUB is a transcription regulatory factor involved in the control of various tick physiological processes including the immune response to pathogen infection and the expression of genes that are important for pathogen infection and multiplication and for tissue structure and function [34,36-39]. SUB is also involved in the regulation of its own expression through the interaction with NF-kB transcription factors that bind to sub core promoter [38]. Therefore, as previously discussed [33,35], the effect of anti-SUB antibodies on sub expression and possibly on SUB function could result in reduction in tick weight and oviposition. The proposed model for SUB function as a protective antigen combines the role of this protein in tick immune response and on the control of other genes necessary for pathogen infection [39]. Targeting SUB by vaccination or RNAi reduces tick immunity, thereby increasing pathogen infection levels. However, lower pathogen infection levels may result from the effect of SUB on tissue structure and function and the expression of genes that 

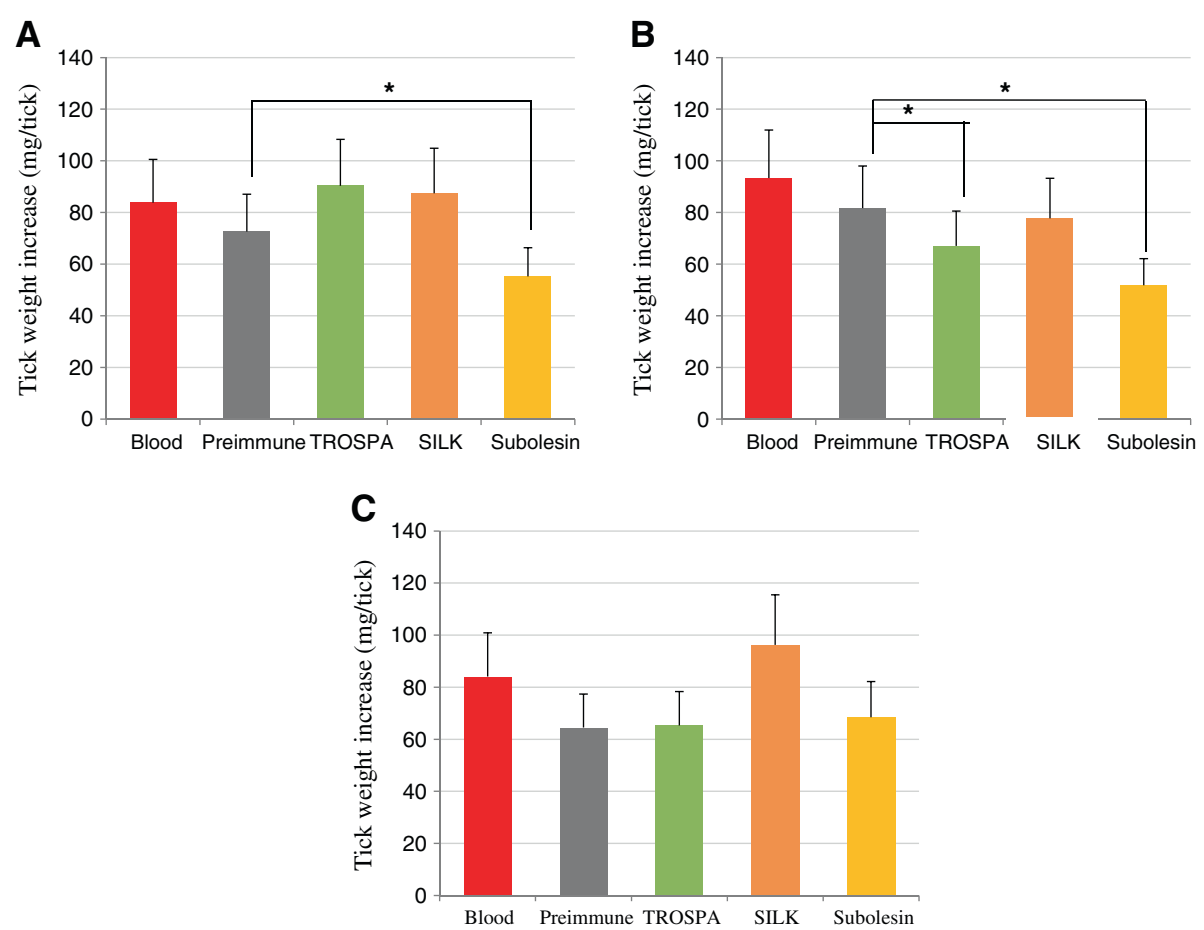

Figure 4 Effect of antibodies on tick weight. (A) Ticks capillary-fed on uninfected blood without (Blood) and with preimmune and anti-tick protein IgGs (TROSPA, SILK and Subolesin). (B) Ticks capillary-fed on B. bigemina-infected blood without (Blood) and with preimmune and antitick protein IgGs. (C) Ticks capillary fed on A. marginale-infected blood without (Blood) and with preimmune and anti-tick protein lgGs. Ticks $(N=15)$ were weighed before and after capillary feeding, the tick weight increase calculated as final minus initial tick weight, expressed as Ave + S.D. (mg/tick) and compared between the group with preimmune antibodies and the other groups by Student's t-test $\left({ }^{*} P<0.05\right)$.

are important for pathogen infection and multiplication [39]. Similarly to ticks fed on SUB-vaccinated cattle [33,35], the ingestion of less infected blood and interference with pathogen infection and multiplication in ticks capillary-fed on blood with anti-SUB antibodies should have resulted in lower pathogen infection levels. However, reduction in tick pathogen DNA levels were not observed in the present study possibly due to different factors. These factors include (a) tick-to-tick variations in infection levels that require the analysis of a larger number of ticks, (b) parasitemia levels in blood used for capillary feeding $(0.7 \%$ IE in the present study) that may need to be higher to evidence the effect of the antibodies on pathogen infection, (c) a non-specific effect of the preimmune serum under this experimental conditions and/or (d) differences in pathogen infection mechanisms between in vivo tick feeding and in vitro capillary feeding. Although Inokuma and Kemp [14] showed that it is possible to infect cattle ticks with $B$. bigemina using capillary feeding with infected blood, Kocan et al. [18] demonstrated that the in vitro capillary feeding system does not reproduce in vivo $A$. marginale infection in ticks.

\section{TROSPA}

TROSPA was first described in I. scapularis as a receptor for Borrelia burgdorferi, showing potential as a vaccine antigen to control bacterial infection in ticks [40,41]. In $I$. scapularis and $R$ annulatus, trospa mRNA levels increased in response to $B$. burgdorferi and B. bigemina infection, respectively $[11,40]$. After gene knockdown by RNAi, $B$. bigemina DNA levels were $83 \%$ and $70 \%$ lower in $R$. annulatus and $R$. microplus, respectively [11]. Considering TROSPA's function as a tick receptor for B. burgdorferi $[40,41]$, these results suggested that while TROSPA is not involved in tick infestation and oviposition, it might be involved in B. bigemina infection and/or multiplication in $R$. microplus. However, in the present study feeding ticks on anti-TROSPA antibodies did not show any effect on pathogen infection, possibly due to some of the factors discussed above for SUB.

\section{SILK}

The flagelliform SILK protein was identified previously in ticks and orb-weaving spider salivary glands [42-44] and was suggested to play a role in $A$. marginale infection and/ or multiplication in $R$. microplus [10]. It was shown that silk mRNA levels increased in response to $A$. marginale infection of $R$. microplus salivary glands and RNAi experiments showed $74 \%$ tick mortality and $63 \%$ reduction in $A$. marginale DNA levels after gene knockdown [10]. Additionally, Mulenga et al. [45] demonstrated that SILK might 

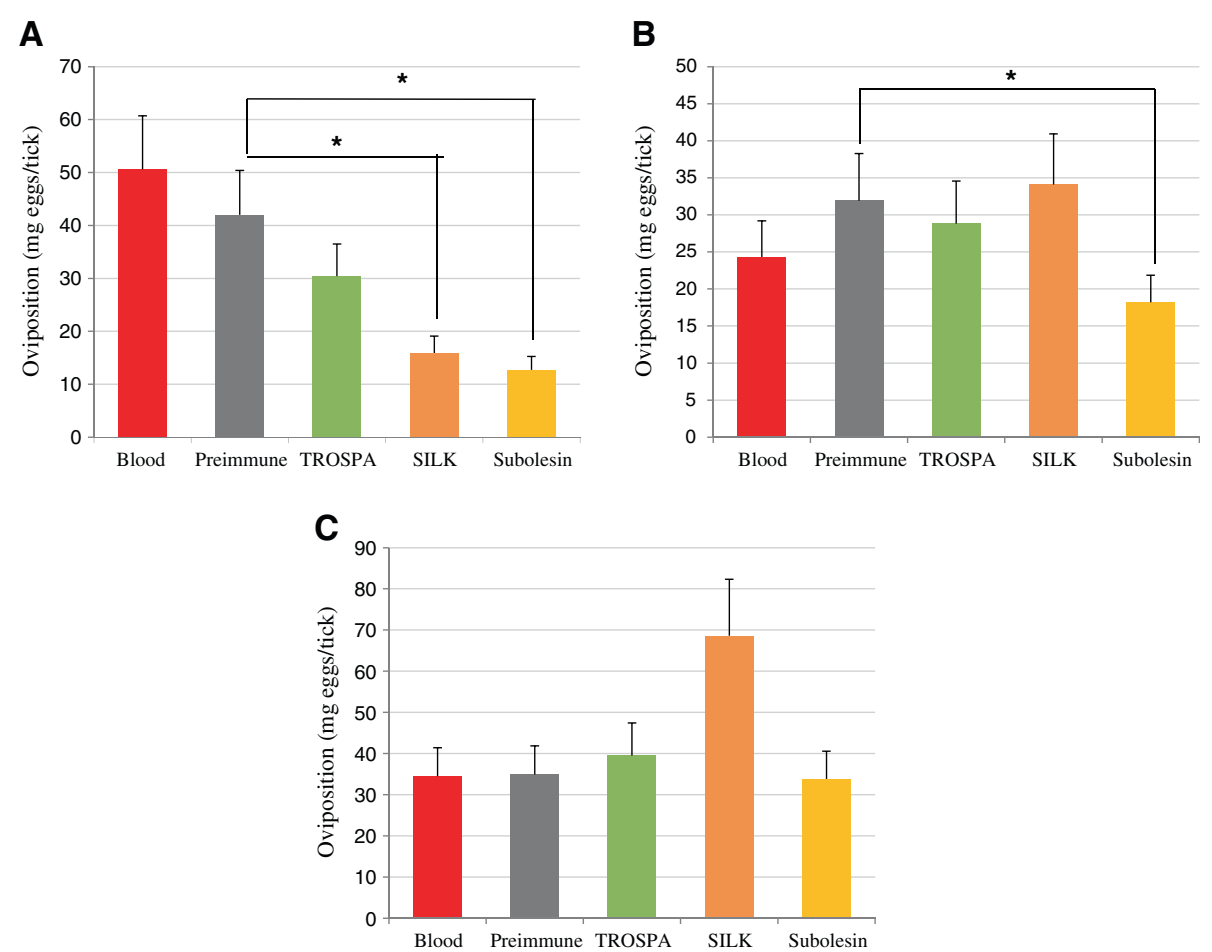

Figure 5 Effect of antibodies on tick oviposition. (A) Ticks capillary-fed on uninfected blood without (Blood) and with preimmune and antitick protein IgGs (TROSPA, SILK and Subolesin). (B) Ticks capillary-fed on B. bigemina-infected blood without (Blood) and with preimmune and anti-tick protein IgGs. (C) Ticks capillary-fed on A. marginale-infected blood without (Blood) and with preimmune and anti-tick protein lgGs. Ticks $(N=5)$ were incubated for oviposition after feeding, the egg mass weight was determined for each tick, expressed as Ave + S.D. (mg eggs/tick) and compared between the group with preimmune antibodies and the other groups by Student's t-test $(* \mathrm{P}<0.05)$.

be involved in tick attachment. In the present study, ticks fed on uninfected blood with anti-SILK antibodies showed decreased oviposition but similar tick weight when compared to ticks fed on preimmune serum, and anti-SILK antibodies did not show any effect on pathogen infection. Therefore, although previous results suggested a role for SILK in tick-host and tick-pathogen interactions, these results were only partially confirmed here.

\section{Conclusions}

The hypothesis behind this experimental approach for the selection of candidate tick protective antigens is that antibodies mediate the main protective mechanism for tick vaccines, something that has been demonstrated for several antigens such as BM86, SUB, SILK and TROSPA for which a direct correlation exists between antibody titers in vaccinated animals and vaccine efficacy [25,35,46-48]. However, if other immune mechanisms are involved in vaccine protection for some antigens, then this experimental approach would not be effective for the selection of protective antigens. The results reported here show that the use of in vitro tick capillary feeding has possibilities and limitations for the characterization of candidate tick protective antigens. Ticks fed by capillary feeding ingested antibodies added to the blood meal and the effect of these antibodies was shown on tick weight and oviposition. However, capillary feeding showed limitations in the study of pathogen infection in ticks possibly associated with different factors such as tick-to-tick variation in infection levels, parasitemia levels in blood used for capillary feeding, a non-specific effect of the preimmune serum and/or differences in pathogen infection between in vivo tick feeding and in vitro capillary feeding, at least for A. marginale. Additionally, the results suggested interactions between antigen-specific antibodies and pathogens in the blood meal that could affect the effect on tick weight and oviposition by still unknown mechanisms. Some of these factors could be optimized for each candidate antigen but would add an additional complexity to this approach. Nevertheless, the combination of RNAi functional studies shown in previous work with tick capillary feeding using antibodies against selected proteins involved in tick-pathogen interactions should allow for a better and more efficient selection of candidate vaccine antigens. The use of dsRNA or siRNA for RNAi has the possibility of inducing off-target effects that could mislead the selection of tick candidate protective antigens [49]. However, off-target effects are sequence-specific and, as shown for sub, could vary between different molecules [50]. As previously discussed [12], RNAi alone is not a suitable tool for the identification 
A

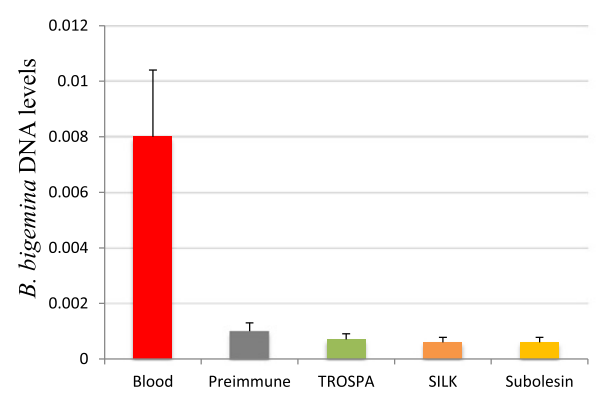

C

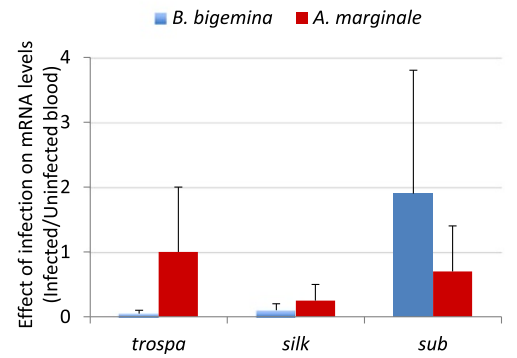

B

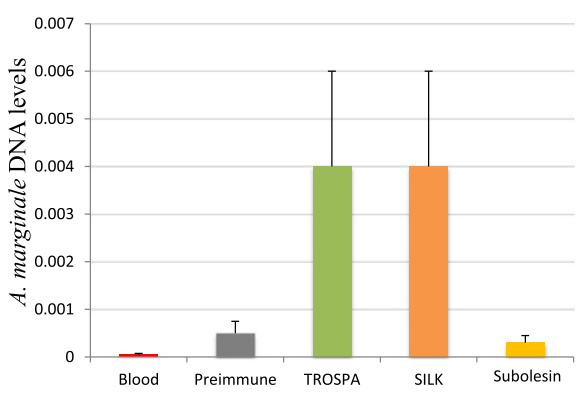

D

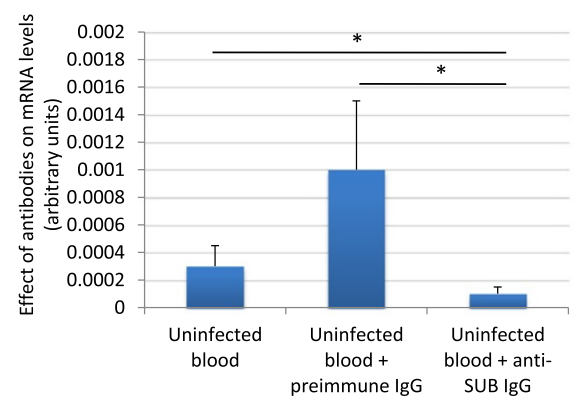

Figure 6 Effect of antibodies on pathogen infection and gene expression. (A) B. bigemina DNA levels were determined by real-time PCR in ticks capillary-fed on B. bigemina-infected blood without (Blood) and with preimmune and anti-tick protein IgGs (TROSPA, SILK and Subolesin). The DNA levels were normalized against tick 165 rDNA, shown as Ave + S.D. normalized Ct values (arbitrary units) and compared between the group with preimmune antibodies and the other groups by Student's t-Test $(P>0.05 ; N=10)$. (B) $A$. marginale DNA levels were determined by real-time PCR in ticks capillary-fed on A. marginale-infected blood without (Blood) and with preimmune and anti-tick protein IgGs. The DNA levels were normalized against tick 165 rDNA, shown as Ave + S.D. normalized Ct values (arbitrary units) and compared between the group with preimmune antibodies and the other groups by Student's t-Test $(P>0.05 ; \mathrm{N}=10)$. (C) Effect of $A$. marginale and B. bigemina infection on gene expression. The mRNA levels of genes encoding for tick proteins trospa, silk and sub were characterized by real-time RT-PCR in ticks capillary-fed on uninfected and infected blood. The mRNA levels were normalized against tick 165 rRNA, shown as the Ave + S.D. infected/uninfected blood Ct ratio (arbitrary units) and compared between ticks fed on infected and uninfected blood by Student's t-Test ( $P>0.05 ; N=10)$. (D) Effect of antiSUB antibodies on sub gene expression. The sub mRNA levels were characterized by real-time RT-PCR in ticks capillary-fed on uninfected blood alone or with the addition of preimmune or anti-SUB IgGs. The mRNA levels were normalized against tick 16S rRNA, shown as Ave + S.D. normalized Ct values (arbitrary units) and compared between groups by Student's t-Test ( ${ }^{*} p \leq 0.05 ; N=10$ ).

of tick protective antigens, but a tool to integrate with other methods such as capillary feeding using antibodies against selected proteins for the identification and functional characterization of candidate tick protective antigens. In the present study, all three selected antigens, SUB, TROSPA and SILK, showed an effect on tick weight and/or oviposition in ticks fed with uninfected or infected blood containing antigen-specific IgGs. These results together with previous results of RNAi experiments suggest that these proteins are good candidate vaccine antigens for the control of $R$. microplus infestations and infection with $A$. marginale and B. bigemina. A recently completed vaccine trial did validate this approach for the selection of candidate tick protective antigens and proved the efficacy of SUB, SILK and TROSPA for the control of $R$. microplus infestations and infection with $A$. marginale and $B$. bigemina [35]. Furthermore, a field trial using the recombinant SUB-MSP1a antigen for vaccination of cattle and sheep showed a reduction in tick infestations and the prevalence for some tick-borne pathogens [51].

\section{Competing interests}

The authors declare that they have no competing interests.

\section{Authors' contributions}

JF and AD designed the study and performed data analysis; OM, SA and JM performed the tick work. OM, SA, JAM-C, LB-S, PA, RF, SW and JMPL performed the laboratory work. JF wrote the manuscript and all authors edited the manuscript. All authors read and approved the final manuscript.

\section{Acknowledgements}

We thank Miguel A García Ortiz (CENID-PAVET, INIFAP, Mexico) for providing the A. marginale strain, Ulrike Munderloh (University of Minnesota) for providing the IDE8 and ISE6 cell lines and Mario Hidalgo Ruiz (University of Queretaro, Mexico) for technical assistance. This research was supported by grants BFU2011-23896, the project PTDC/CVT/112050/2009 funded by the Fundação para a Ciência e Tecnologia (FCT) and the EU FP7 ANTIGONE project number 278976. O. Merino and S. Weisheit are Early Stage Researchers supported by the POSTICK ITN (Post-graduate training network for capacity building to control ticks and tick-borne diseases) within the 
FP7-PEOPLE-ITN programme (EU Grant No. 238511). S. Antunes is the recipient of a PhD grant supported by the FCT (SFRH/BD/48251/2008).

\section{Author details}

${ }^{1}$ Instituto de Higiene e Medicina Tropical, Rua da Junqueira 100, 1349-008 Lisboa, Portugal. ${ }^{2}$ SaBio. Instituto de Investigación en Recursos Cinegéticos IREC-CSIC-UCLM-JCCM, Ronda de Toledo s/n, 13005 Ciudad Real, Spain. ${ }^{3}$ Facultad de Ciencias Naturales, Universidad Autónoma de Querétaro, Campus Juriquilla CP 76230, Querétaro, Mexico. ${ }^{4}$ The Roslin Institute, University of Edinburgh, Easter Bush, Midlothian EH25 9RG UK. ${ }^{5}$ The Pirbright Institute, Ash Road, Pirbright, Woking GU24 ONF, UK. ${ }^{6}$ Centro de Malária e Outras Doenças Tropicais, Instituto de Higiene e Medicina Tropical, Rua da Junqueira 100, 1349-008 Lisboa, Portugal. ${ }^{7}$ Department of Veterinary Pathobiology, Center for Veterinary Health Sciences, Oklahoma State University, Stillwater, OK 74078, USA.

Received: 30 December 2013 Accepted: 19 January 2014

Published: 22 January 2014

\section{References}

1. Jongejan F, Uilenberg G: The global importance of ticks. Parasitology 2004, 129:S3-S14.

2. Estrada-Peña A, Bouattour A, Camicas JL, Guglielmone A, Horak I, Jongejan F, Latif A, Pegram R, Walker AR: The known distribution and ecological preferences of the tick subgenus Boophilus (Acari: Ixodidae) in Africa and Latin America. Exp Appl Acarol 2006, 38:219-235.

3. Peter RJ, Van den Bossche P, Penzhorn BL, Sharp B: Tick, fly, and mosquito control- lessons from the past, solutions for the future. Vet Parasitol 2005, 132:205-215.

4. Graf JF, Gogolewski R, Leach-Bing N, Sabatini GA, Molento MB, Bordin EL, Arantes GJ: Tick control: an industry point of view. Parasitology 2004, 129:S427-S442.

5. de la Fuente J: Vaccines for vector control: exciting possibilities for the future. Vet J 2012, 194:139-140

6. de la Fuente J, Almazán C, Canales M, Pérez de la Lastra JM, Kocan KM, Willadsen P: A ten-year review of commercial vaccine performance for control of tick infestations on cattle. Anim Health Res Rev 2007, 8:23-28,

7. Almazán C, Lagunes R, Villar M, Canales M, Rosario-Cruz R, Jongejan F, de la Fuente J: Identification and characterization of Rhipicephalus (Boophilus) microplus candidate protective antigens for the control of cattle tick infestations. Parasitol Res 2010, 106:471-479.

8. de la Fuente J, Blouin EF, Manzano-Roman R, Naranjo V, Almazán C, Pérez de la Lastra JM, Zivkovic Z, Jongejan F, Kocan KM: Functional genomic studies of tick cells in response to infection with the cattle pathogen Anaplasma marginale. Genomics 2007, 90:712-722.

9. Zivkovic Z, Torina A, Mitra R, Alongi A, Scimeca S, Kocan KM, Galindo RC, Almazán C, Blouin EF, Villar M, Nijhof AM, Mani R, la Barbera G, Caracappa S, Jongejan $\mathrm{F}$, de la Fuente J: Subolesin expression in response to pathogen infection in ticks. BMC Immunol 2010, 11:7.

10. Zivkovic Z, Esteves E, Almazán C, Daffre S, Nijhof AM, Kocan KM, Jongejan F, de la Fuente J: Differential expression of genes in salivary glands of male Rhipicephalus (Boophilus) microplus in response to infection with Anaplasma marginale. BMC Genomics 2010, 11:186

11. Antunes S, Galindo R, Almazan C, Rudenko N, Golovchenko M, Grubhoffer L, Shkap V, do Rosário V, de la Fuente J, Domingos A: Functional genomics studies of Rhipicephalus (Boophilus) annulatus ticks in response to infection with the cattle protozoan parasite, Babesia bigemina. Int J Parasitol 2012, 42:187-195.

12. de la Fuente J, Merino O: Vaccinomics, the new road to tick vaccines. Vaccine 2013, 31:5923-5929.

13. Rau U, Hannoun C: The use of a capillary-tube technique for artificially feeding Argas reflexus reflexus ticks. Bull World Health Organ 1968, 39:332-333.

14. Inokuma H, Kemp DH: Establishment of Boophilus microplus infected with Babesia bigemina by using in vitro tube feeding technique. J Vet Med Sci 1998, 60:509-512.

15. Macaluso KR, Sonenshine DE, Ceraul SM, Azad AF: Infection and transovarial transmission of rickettsiae in Dermacentor variabilis ticks acquired by artificial feeding. Vector Borne Zoonotic Dis 2001, 1:45-53.

16. Sonenshine DE, Ceraul S, Hynes W, Macaluso KR, Azad AF: Expression of defensin-like peptides in tick hemolymph and midgut in response to challenge with Borrelia burgdorferi, Escherichia coli and Bacillus subtilis Exp Appl Acarol 2002, 28:127-134

17. de la Fuente J, Almazán C, Van Den Busche RA, Bowman J, Yoshioka JH, Kocan KM: Characterization of genetic diversity in two populations of Dermacentor andersoni (Acari: Ixodidae). Exp Parasitol 2005, 109:16-26.

18. Kocan KM, Yoshioka J, Sonenshine DE, de la Fuente J, Ceraul SM, Blouin EF, Almazán C: Capillary tube feeding system for studying tick-pathogen interactions of Dermacentor variabilis (Acari: Ixodidae) and Anaplasma marginale (Rickettsiales: Anaplasmataceae). J Med Entomol 2005, 42:864-874

19. Kröber T, Guerin PM: In vitro feeding assays for hard ticks. Trends Parasitol 2007, 23:445-449.

20. Matsuo T, Inoue N, Ruheta MR, Taylor D, Fujisaki K: Tickcidal effect of monoclonal antibodies against hemocytes, $0 \mathrm{~m} 21$, in an adult female tick, Ornithodoros moubata (Acari: Argasidae). J Parasitol 2004, 90:715-720

21. Almazán C, Blas-Machado U, Kocan KM, Yoshioka J, Blouin EF, Mangold AJ, de la Fuente J: Characterization of three Ixodes scapularis cDNAs protective against tick infestations. Vaccine 2005, 23:4403-4416.

22. Gonsioroski AV, Bezerra IA, Utiumi KU, Driemeier D, Farias SE, da Silva Vaz I, Masuda A: Anti-tick monoclonal antibody applied by artificial capillary feeding in Rhipicephalus (Boophilus) microplus females. Exp Parasitol 2012, 130:359-363.

23. Fragoso-Sanchez H, Garcia-Vazquez Z, Tapia-Perez G, Ortiz-Najera A, RosarioCruz R, Rodriguez-Vivas I: Response of Mexican Rhipicephalus (Boophilus) microplus ticks to selection by amitraz and genetic analysis of attained resistance. J Entomol 2011, 8:218-228.

24. Mosqueda J, Ramos JA, Falcon A, Alvarez JA, Aragon V, Figueroa JV: Babesia bigemina: Sporozoite isolation from Boophilus microplus nymphs and initial immunomolecular characterization. Ann N Y Acad Sci 2004, 1026:222-231

25. Merino O, Almazán C, Canales M, Villar M, Moreno-Cid JA, Estrada-Peña A Kocan KM, de la Fuente J: Control of Rhipicephalus (Boophilus) microplus infestations by the combination of subolesin vaccination and tick autocidal control after subolesin gene knockdown in ticks fed on cattle. Vaccine 2011, 29:2248-2254.

26. Bell-Sakyi L: Ehrlichia ruminantium grows in cell lines from four ixodid tick genera. J Comp Pathol 2004, 130:285-293.

27. Munderloh UG, Liu Y, Wang M, Chen C, Kurtti TJ: Establishment, maintenance and description of cell lines from the tick Ixodes scapularis. J Parasitol 1994, 80:533-543.

28. Kurtti TJ, Munderloh UG, Andreadis TG, Magnarelli LA, Mather TN: Tick cell culture isolation of an intracellular prokaryote from the tick lxodes scapularis. J Inv Path 1996, 67:318-321.

29. Munderloh UG, Kurtti TJ: Formulation of medium for tick cell culture. Exp Appl Acarol 1989, 7:219-229.

30. Rodríquez-Hernández E, Mosqueda J, Álvarez-Sánchez ME, Falcón Neri A Mendoza-Hernández G, Camacho-Nuez M: The identification of a VDAClike protein involved in the interaction of Babesia bigemina sexual stages with Rhipicephalus microplus midgut cells. Vet Parasitol 2012, 187:538-541.

31. Ocampo Espinoza V, Vázquez JE, Aguilar MD, Ortiz MA, Alarcón GJ, Rodríquez SD: Anaplasma marginale: lack of cross-protection between strains that share MSP1a variable region and MSP4. Vet Microbiol 2006, 114:34-40.

32. Nijhof AM, Taoufik A, de la Fuente J, Kocan KM, de Vries E, Jongejan F: Gene silencing of the tick protective antigens, Bm86, Bm91 and subolesin, in the one-host tick Boophilus microplus by RNA interference. Int J Parasitol 2007, 37:653-662

33. Merino O, Almazán C, Canales M, Villar M, Moreno-Cid JA, Galindo RC, de la Fuente J: Targeting the tick protective antigen subolesin reduces vector infestations and pathogen infection by Anaplasma marginale and Babesia bigemina. Vaccine 2011, 29:8575-8579.

34. de la Fuente J, Almazán C, Blas-Machado U, Naranjo V, Mangold AJ, Blouin EF, Gortazar C, Kocan KM: The tick protective antigen, 4D8, is a conserved protein involved in modulation of tick blood ingestion and reproduction. Vaccine 2006, 24:4082-4095.

35. Merino M, Antunes S, Mosqueda J, Moreno-Cid JA, Pérez de la Lastra JM, Rosario-Cruz R, Rodríguez S, Domingos A, de la Fuente J: Vaccination with proteins involved in tick-pathogen interactions reduces vector infestations and pathogen infection. Vaccine 2013, 31:5889-5896.

36. de la Fuente J, Moreno-Cid JA, Canales M, Villar M, Pérez de la Lastra JM, Kocan KM, Galindo RC, Almazán C, Blouin EF: Targeting arthropod 
subolesin/akirin for the development of a universal vaccine for control of vector infestations and pathogen transmission. Vet Parasitol 2011, 181:17-22.

37. Hajdusek O, Šíma R, Ayllón N, Jalovecká M, Perner J, de la Fuente J, Kopáček $P$ : Interaction of the tick immune system with transmitted pathogens. Front Cell Infect Microbiol 2013, 3:26.

38. Naranjo N, Ayllón N, de la Lastra JM P, Galindo RC, Kocan KM, Blouin EF, Mitra R, Alberdi P, Villar M, de la Fuente J: Reciprocal regulation of NF-kB (Relish) and Subolesin in the tick vector, Ixodes scapularis. PLOS ONE 2013, 8:e65915.

39. Merino O, Alberdi P, Pérez de la Lastra JM, de la Fuente J: Tick vaccines and the control of tick-borne pathogens. Front Cell Infect Microbiol 2013, 3:30.

40. Pal U, Li X, Wang T, Montgomery RR, Ramamoorthi N, Desilva AM, Bao F, Yang X, Pypaert M, Pradhan D, Kantor FS, Telford S, Anderson JF, Fikrig E: TROSPA, an Ixodes scapularis receptor for Borrelia burgdorferi. Cell 2004, 119:457-468.

41. Hovius JW, van Dam AP, Fikrig E: Tick-host-pathogen interactions in Lyme borreliosis. Trends Parasitol 2007, 23:434-438.

42. Hayashi CY, Lewis RV: Molecular architecture and evolution of a modular spider silk protein gene. Science 2000, 287:1477-1479.

43. Alarcon-Chaidez FJ, Sun J, Wikel SK: Transcriptome analysis of the salivary glands of Dermacentor andersoni Stiles (Acari: Ixodidae). Insect Biochem Mol Biol 2007, 37:48-71.

44. Santos IK, Valenzuela JG, Ribeiro JM, de Castro M, Costa JN, Costa AM, da Silva ER, Neto OB, Rocha C, Daffre S, Ferreira BR, da Silva JS, Szabó MP, Bechara GH: Gene discovery in Boophilus microplus, the cattle tick: the transcriptomes of ovaries, salivary glands, and hemocytes. Ann N Y Acad Sci 2004, 1026:242-246.

45. Mulenga A, Blandon M, Khumthong R: The molecular basis of the Amblyomma americanum tick attachment phase. Exp Appl Acarol 2007 , 41:267-287.

46. de la Fuente J, Rodríguez M, Redondo M, Montero C, García-García JC, Méndez L, Serrano E, Valdés M, Enríquez A, Canales M, Ramos E, de Armas CA, Rey S, Rodríguez JL, Artiles M, García L: Field studies and costeffectiveness analysis of vaccination with Gavac ${ }^{T M}$ against the cattle tick Boophilus microplus. Vaccine 1998, 16:366-373.

47. Popara M, Villar M, Mateos-Hernández L, de Mera IG F, Marina A, del Valle M, Almazán C, Domingos A, de la Fuente J: Lesser protein degradation machinery correlates with higher BM86 tick vaccine efficacy in Rhipicephalus annulatus when compared to R. microplus. Vaccine 2013, 31:4728-4735.

48. Moreno-Cid JA, de la Lastra JM P, Villar M, Jiménez M, Pinal R, Estrada-Peña A, Alarcón P, Delacour S, Oropeza V, Ruiz I, Molina R, Lucientes J, Prudencio CR, Galindo RC, Almazán C, Nijhof AM, Mangold AJ, Gortázar C, de la Fuente $\mathrm{J}$ : Control of multiple arthropod vector infestations with subolesin/akirin vaccines. Vaccine 2013, 31:1187-1196.

49. Lew-Tabor AE, Kurscheid S, Barrero R, Gondro C, Moolhuiizen PM, Rodriguez Valle M, Morgan JA, Covacin C, Bellgard MI: Gene expression evidence for off-target effects caused by RNA interference-mediated gene silencing of Ubiquitin-63E in the cattle tick Rhipicephalus microplus. Int J Parasitol 2011, 41:1001-1014.

50. de la Fuente J, Maritz-Olivier C, Naranjo V, Ayoubi P, Nijhof AM, Almazán C, Canales M, de la Lastra JM P, Galindo RC, Blouin EF, Gortazar C, Jongejan F, Kocan KM: Evidence of the role of tick subolesin in gene expression. BMC Genomics 2008, 9:372.

51. Torina A, Moreno-Cid JA, Blanda V, de Mera IG F, de la Lastra JM P, Scimeca S, Blanda M, Scariano ME, Briganò S, Disclafani R, Piazza A, Vicente J, Gortázar C, Caracappa S, Lelli RC, de la Fuente J: Control of tick infestations and pathogen prevalence in cattle and sheep farms vaccinated with the recombinant Subolesin-Major Surface Protein 1a chimeric antigen. Parasites Vectors 2014, 7:10.

doi:10.1186/1756-3305-7-42

Cite this article as: Antunes et al: Tick capillary feeding for the study of proteins involved in tick-pathogen interactions as potential antigens for the control of tick infestation and pathogen infection. Parasites \& Vectors 2014 7:42.

\section{Submit your next manuscript to BioMed Central and take full advantage of:}

- Convenient online submission

- Thorough peer review

- No space constraints or color figure charges

- Immediate publication on acceptance

- Inclusion in PubMed, CAS, Scopus and Google Scholar

- Research which is freely available for redistribution 